\title{
Photometric Stereo with Area Lights for Lambertian Surfaces
}

\author{
Jiangbin Gan \\ ganjiangbin@informatik.uni-marburg.de \\ University of Marburg \\ Marburg, Germany
}

\author{
Thorsten Thormählen \\ thormae@informatik.uni-marburg.de \\ University of Marburg \\ Marburg, Germany
}

\begin{abstract}
This paper presents a photometric stereo technique that uses area lights for normal recovery and 3D geometry reconstruction of midsized objects. The object is illuminated in succession by several off-the-shelf LED area lights and images are captured by at least two DSLR cameras. Compared to point light sources, area lights have the advantage of producing high illuminance, resulting in low image noise and fast shutter speed, which is important if the captured object is not completely static during the acquisition of the images, e.g., when capturing a human face. Area lights are standard photo equipment which makes them cheaper, easier to obtain, and install than specialized many-lights hardware. The normal map of the object is recovered by our photometric stereo approach that uses ray tracing techniques to simulate the light transport in the scene. Furthermore, our approach takes the effects of occlusion and interreflections into account. The normal map is iteratively optimized which in turn is utilized to update the depth information of the object. Our synthetic and real-world experiments show that area lights are applicable for photometric stereo at the cost of an increased computational effort.
\end{abstract}

\section{CCS CONCEPTS}

- Computing methodologies $\rightarrow$ Reconstruction.

\section{KEYWORDS}

photometric stereo, reconstruction, area light, normal map

\section{ACM Reference Format:}

Jiangbin Gan and Thorsten Thormählen. 2021. Photometric Stereo with Area Lights for Lambertian Surfaces. In European Conference on Visual Media Production (CVMP '21), December 6-7, 2021, London, United Kingdom. ACM, New York, NY, USA, 7 pages. https://doi.org/10.1145/3485441.3485651

\section{INTRODUCTION}

After photometric stereo was introduced by Woodham [Woodham 1980] in 1980, many approaches have been proposed for the recovery of surface normals from images taken with varying illumination conditions. Traditionally, photometric stereo is performed under the assumption that the light source is located at infinity. However, in practice, a point light source cannot be an ideal directional light. The directional light model ignores the varying incident light direction and distance from a point light to different surface locations on the inspected object. In addition, the effects of occlusion and

ACM acknowledges that this contribution was authored or co-authored by an employee contractor or affiliate of a national government. As such, the Government retains a nonexclusive, royalty-free right to publish or reproduce this article, or to allow others to do so, for Government purposes only.

CVMP '21, December 6-7, 2021, London, United Kingdom

(C) 2021 Association for Computing Machinery.

ACM ISBN 978-1-4503-9094-1/21/12 . \$ \$15.00

https://doi.org/10.1145/3485441.3485651 interreflections are often ignored in traditional approaches. These three simplifying assumptions (i.e., directional light sources, no occlusion, and no interreflections) are the main sources of error for the generation of a normal map.

Instead of using a few point lights, some approaches utilize many light sources to generate the desired illumination pattern, such as distributed light sources [Clark 2010] and the light stage setups [Debevec 2012]. One of the main advantages of using a many-lights approach is that a larger number of lights can produce an overall higher illuminance. A high illuminance corresponds to a reduced acquisition time and/or reduced signal-to-noise ratios for the captured images [Schechner et al. 2003].

However, despite these benefits, many-lights systems also have their own inherent problems. In the presence of a large number of lights, the occlusion and interreflections can get worse. With many lights the likelihood that at least some of them are occluded is high. For non-convex surfaces, the advantage that multiple lights produce a high illuminance for surface patches might increase the problem of interreflections received from adjacent visible patches. Although the effects of occlusion and interreflections become more significant in many-light systems, almost all existing approaches that use multiple light sources lack solutions to deal with them. Moreover, these systems usually require special hardware that is relatively difficult to build and transport to different locations.

In this paper, we propose a photometric stereo approach using off-the-shelf LED area lights. We first employ an illumination model for area lights to compute an initial 3D reconstruction. The initial $3 \mathrm{D}$ information is used to compute the geometric relation between the area light and the surface points. We iteratively optimize the normal map and integrate the depth information which is in turn used to refine the normal map. Furthermore, a ray tracing technique is applied to compute errors by occlusion and interreflections. The main contributions of this paper are:

(1) Our illumination setup is built with off-the-shelf LED area lights. It is easy to obtain, build, transport, and expand.

(2) We present an approach that allows to iteratively compute a normal map from multiple area lights.

(3) Compared to previous many-lights photometric stereo approaches, we explicitly take nearby light sources into account.

(4) Our approach reduces the errors introduced by occlusion and interreflections.

\section{RELATED WORK}

Photometric Stereo. Most traditional photometric stereo approaches use the assumption of ideal directional lighting. The assumption typically fails in reality when the lights are close to the observed objects. On the other hand, many recent studies focus on dealing with nearby lighting. Logothetis et al. [Logothetis et al. 2017] 
proposed a variational approach for 3D reconstruction with the prior knowledge of the positions of the light sources. Xie et al.[Xie et al. 2019] developed a computational framework to solve the non-linear problem of nearby lighting through iterative mesh deformations. Liu et al. [Liu et al. 2018] proposed a two-stage algorithm for nearby photometric stereo by using a planar circular ring of LED light sources which is centered around the camera lens. Santo at el. [Santo et al. 2020] considered nearby lighting with spatially varying reflectances using deep neural networks. In [Quéau et al. 2018] Quéau at el. use reweighted least-squares to solve a PDE system for depth recovery. There are areas where the shape and the albedo are poorly estimated because the area is not well illuminated. This potential problem of point light sources can be largely avoided by using area light sources. Clark [Clark 2006, 2010] considered the problem of shape-from-shading by using a rectangular planar nearby distributed uniform isotropic illuminant. The illuminant can be seen as a single isotropic point light source at infinity in the absence of shadowing. Thus, the classical photometric stereo algorithm can be employed. Although nearby lighting is taken into account, self-shadowing and mutual illumination are ignored.

Photometric Stereo with Area Lights. In 1981, Ikeuchi [Ikeuchi 1981] proposed a shape-from-shading approach which is perhaps the first to make use of a distributed light source to recover surface orientation of specular materials. Schechner et al. [Schechner et al. 2003] proposed a multiplexed illumination approach, in which the object is illuminated by many light sources simultaneously using a multiplexing code. The acquired images need to be demultiplexed on a computer afterwards. Funk and Yang [Funk and Yang 2007] used a raster display device as a controlled lighting source. Six squared areas on the display are used as light sources to provide multiple illuminations for photometric stereo. Light attenuation and self-occlusion are not considered. Quéau at el. [Quéau et al 2016] employed a matte screen to display a collection of images for photometric stereo. Hertzmann and Seitz [Hertzmann and Seitz 2005] proposed an approach to recover the surface shape of objects with unknown reflectance properties. It is mentioned that the algorithms can be extended to area light sources but the experiments are only performed with point light sources. Similarly, in [Liao et al. 2011], Liao et al. proposed a photometric stereo approach, which can be extended to area lights, but the paper contains no corresponding experimental data. Song at el. [Song et al. 2020] proposed a hybrid $3 \mathrm{D}$ reconstruction approach by combing the absolute depth information from stripe-based structured light (SL) with photometric stereo using area lights. Santo at el. [Santo et al. 2020] proposed a learning-based photometric stereo method. Currently, this approach is not suitable for high-quality reconstructions because the image resolution is very restricted.

In contrast to many previous approaches, our proposed solution takes the area light geometry, distance, and light attenuation into account. We further explicitly deal with occlusion and interreflections by using ray tracing on the initial reconstruction generated from normal integration.

Many-lights Photometric Stereo. Probably the most well-known approach for capturing surface normals using specialized setups is the light stage [Debevec 2012; Ma et al. 2007; Wilson et al. 2010]. It employs a lighting sphere as an acquisition hardware to capture the object under spherical gradient illuminations. The gradient illumination leads to a simple equation for computing surface normals, which can be implemented by fast image processing operations. Because many light sources are employed, the total illuminance is high, which results in low image noise and allows for a fast shutter speed of the cameras.

While the light stage's spherical gradient illumination provides convincing surface normals for movie production and visual effects, the estimates are usually biased because the effects of occlusion and interreflections are not considered.

With its many custom hardware elements, a light stage setup is usually difficult to built and move to different locations. Nevertheless, in 2014, a mobile version of the light stage was constructed by Debevec and coworkers to capture the face of President Obama at the White House.

\section{IMAGE ACQUISITION SETUP}

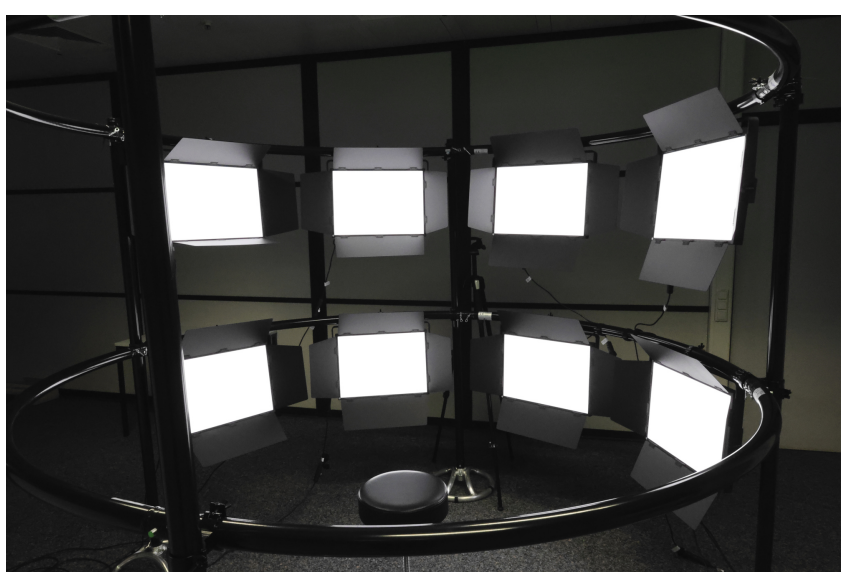

Figure 1: Our illumination setup consists of eight off-theshelf area lights that are mounted on a ring-shaped truss

Our image acquisition hardware is shown in Figure 1. It aims at recovering 3D information (i.e., surface normals and depth information) for mid-sized objects, such as human faces.

The setup consists of eight large LED area light sources and at least two digital SLR camera. Each area light is internally built from equidistantly arranged LED lights, has a size of $54.5 \times 50 \mathrm{~cm}$, and can produce a luminous flux of up to 5000 lumen. The individual LEDs are covered by an optical diffuser such that they produce an approximately uniform luminance.

The eight area lights are mounted on a standard ring-shaped truss with a diameter of 2.5 meters. In practice, the positions of the lights must be chosen such that each surface point of the object is illuminated by at least three lights.

The complete hardware is built from standard components and is easy to disassemble and assemble at different locations.

During image acquisition, the area lights are turned on and off one by one and for each illumination direction the cameras are triggered. Thus, eight input images are obtained for each camera. When an area light is on, all its LEDs are on. The LEDs do not need to be controlled individually. 




Figure 2: Images of a human face captured with our acquisition hardware

The locations of the area lights are calibrated manually by measuring the positions of three corners of the casing with a laser telemeter. Thereby, the origin of the reference coordinate system is placed exactly in the center of the ring-shaped truss. The intrinsic and extrinsic camera parameters are estimated by a camera calibration technique that uses multiple calibration patterns [Grochulla et al. 2011]. The camera calibration is performed in the same coordinate system as the lights (center of the ring-shaped truss).

Figure 2 shows the input images for a human face illuminated subsequently by the eight area light.

\section{INITIAL NORMAL MAP ESTIMATION}

Traditional photometric stereo assumes that a light is placed at infinity. Considering the case of a Lambertian surface illuminated by a perfect directional light source with unit illuminance, the luminance at pixel $(x, y)$ on the image $L$ can be denoted by:

$$
L(x, y)=\frac{\rho}{\pi} \mathbf{n}^{\top} \mathbf{1}
$$

where $\rho$ is the diffuse albedo of the object, 1 is the direction of the incident light, $\mathbf{n}$ is the unknown normal at pixel $(x, y)$, and $\mathbf{n}^{\top} \mathbf{l}$ denotes the dot product.

If we consider a real area light instead of a light at infinity, the light transport is more complex. It can be expressed using the rendering equation in area form:

$$
L(x, y)=\int_{\mathcal{A}} \mathrm{f}_{r} L_{A}(\mathbf{l}) \frac{\left(\mathbf{n}^{\top} \mathbf{l}\right)\left(-\mathbf{n}_{A}^{\top} \mathbf{l}\right)}{r^{2}} d A,
$$

where $f_{r}$ is the $\mathrm{BRDF}$ of the surface, $L_{A}(\mathbf{l})$ is the incident luminance from direction $\mathbf{l}, \mathcal{A}$ is the domain of the area light, $\mathbf{n}_{A}$ is its normal direction, and $r$ is the distance from the observed surface point to the infinitesimal $d A$ on the light. If we assume a Lambertian surface, the BRDF is constant. Furthermore, if the area light is an Lambertian emitter, its luminance $L_{A}$ is constant:

$$
L(x, y)=\frac{\rho}{\pi} L_{A} \int_{\mathcal{A}} \frac{\left(\mathbf{n}^{\top} \mathbf{l}\right)\left(-\mathbf{n}_{A}^{\top} \mathbf{l}\right)}{r^{2}} d A .
$$

The integral can be approximated by sampling. In practice, the area light usually consists of uniformly arranged LEDs, which we used as sampling points. However, more (or less) sampling points can be used as well. Provided that the distribution of the sampling points is uniform for the complete domain, the integral can be computed by

$$
L(x, y) \approx \frac{\rho}{\pi} L_{A} \frac{A}{K} \sum_{k=1}^{K} \frac{\left(\mathbf{n}^{\top} \mathbf{l}_{k}\right)\left(-\mathbf{n}_{A}^{\top} \mathbf{l}_{k}\right)}{r_{k}^{2}},
$$

where $A$ is the total area of the light and $K$ is the number of sampling points. The directions $\mathbf{l}_{k}$ and distances $r_{k}$ can be computed from the position of each sampling point on the light and the position of the current surface point. However, because the position on the surface is unknown, as a first approximation, we assume that the surface is located at the origin of the reference coordinate system (corresponding to the center of the ring-shaped truss for our image acquisition setup). This initial solution will be refined later. In order for this to work, the captured object must be placed close to the origin of the reference coordinate system.

The unknown normal $\mathbf{n}$ does not depend on $k$ and can be moved in front of the sum

$$
L(x, y) \approx \underbrace{\frac{\rho}{\pi} L_{A}}_{\alpha} \mathbf{n}^{\top} \underbrace{\frac{A}{K} \sum_{k=1}^{K} \frac{\left(-\mathbf{n}_{A}^{\top} \mathbf{l}_{k}\right)}{r_{k}^{2}} \mathbf{l}_{k}}_{\mathrm{G}},
$$

where $\mathrm{G}$ is a 3-vector and we get a different $\mathrm{G}_{g}$ for each area light illumination. With a minimum of three images $L_{g}(x, y)$ taken under the illumination of the different area lights, we get an system of linear equations that we can solve for the three unknowns $\alpha \mathbf{n}=$ $\left(\alpha n_{x}, \alpha n_{y}, \alpha n_{z}\right)^{\top}=\left(u_{x}, u_{y}, u_{z}\right)^{\top}$. To remove $\alpha$, we can use the non-linear constraint that the length of $\mathbf{n}$ is 1.0

$$
\mathbf{n}=\frac{\left(u_{x}, u_{y}, u_{z}\right)^{\top}}{\left|\left(u_{x}, u_{y}, u_{z}\right)\right|} .
$$

In all of our experiments, eight area lights, i.e., eight $\mathrm{G}_{g}$ and corresponding $L_{g}(x, y)$ are utilized to obtain an over-determined system of linear equations.

\section{ITERATIVE OPTIMIZATION}

In this section, we introduce our iterative process for optimizing the normal map based on the initial estimate. To improve the initial normal map, 3D information about the captured object must be taken into account.

An initial 3D reconstruction can be computed by integrating the normals over the image domain. Thereby, each pixel corresponds to a quad (or two triangles) in the resulting 3D mesh. However, because normals are the derivatives of $3 \mathrm{D}$ positions, the reconstruction has an undefined global scaling factor. This unknown scaling factor can be found by back-projecting the 3D mesh into the second camera view. We use the scaling factor that produces the smallest backprojection error.

With the resulting initial 3D reconstruction, an improved normal map can be computed. Firstly, the assumption that the surface is located at the origin of the reference coordinate system is no longer necessary. Instead, the corresponding position on the 3D mesh can be used to compute $r_{k}^{2}$ and $\mathbf{l}_{k}$ in Eq. (5). Secondly, the mesh 
allows computing a visibility $V$ between the sampling point on the area light and the surface point. The visibility term $V$ is 0 if any triangle of the mesh is intersecting the ray between the sampling point and the surface point, and 1 otherwise. Thirdly, we can take interreflections into account. To this end, the equations from the previous section are extended by an additional term for indirect light. Considering these changes, Eq. (2) becomes

$$
\begin{aligned}
L(x, y)= & \underbrace{\int_{\mathcal{A}} \mathrm{f}_{r} L_{A}(\mathbf{l}) \frac{\left(\mathbf{n}^{\top} \mathbf{l}\right)\left(-\mathbf{n}_{A}^{\top} \mathbf{l}\right)}{r^{2}} V(\mathbf{l}) d A}_{\text {area light }} \\
+ & \underbrace{\int_{\Omega(\mathbf{n})} f_{r} L_{i}\left(\mathbf{n}^{\top} \mathbf{l}\right) d \omega}_{\text {indirect light }} .
\end{aligned}
$$

This integral equation is the typical problem solved by a backward path tracer. The direct light is computed by integrating over the area of the light source considering the visibility $V$. The indirect light is computed by integrating over the solid angle $\omega$ of the hemisphere $\Omega(\mathbf{n})$ defined by $\mathbf{n}$.

By uniformly sampling the hemisphere and the area light, we get:

$$
L(x, y) \approx \frac{\rho}{\pi} \frac{A}{K} \sum_{k=1}^{K} L_{A} \frac{\left(\mathbf{n}^{\top} \mathbf{l}_{k}\right)\left(-\mathbf{n}_{A}^{\top} \mathbf{l}_{k}\right)}{r_{k}^{2}} V_{k}+\frac{\rho}{\pi} \frac{2 \pi}{I} \sum_{i=1}^{I} L_{i}\left(\mathbf{n}^{\top} \mathbf{l}_{i}\right)
$$

In our experiments, the ray intersection with the mesh is performed by ray tracing. When the ray tracer reports a hit for the indirect ray direction $\mathrm{l}_{i}$, the corresponding luminance $L_{i}$ can be simply determined by projecting the hit point into the image. For the indirect term, contributions from the area lights are ignored. The ray tracing does not perform multiple bounces, i.e., it stops after a the first indirection. The visibility $V_{k}$ can be determined by shooting shadow rays in the direction $\mathbf{l}_{k}$ to the sampling position on the area light source.

Consequently, the updated $\mathrm{G}$ for Eq. (5) is given by:

$$
\mathrm{G}=\frac{A}{K} \sum_{k=1}^{K} \frac{\left(-\mathbf{n}_{A}^{\top} \mathbf{l}_{k}\right)}{r_{k}^{2}} \mathbf{l}_{k} V_{k}+\frac{2 \pi}{I} \sum_{i=1}^{I} \frac{L_{i}}{L_{A}} \mathbf{l}_{i}
$$

Using the updated $\mathrm{G}$, a new normal map can be computed, as described before. This process can be repeated until convergence.

\section{RESULTS}

To evaluate the proposed approach, we use both synthetic and real-world data in our experiments. For comparison, we employ the traditional point light photometric stereo approach [Woodham 1980] from Eq. (1) (referred to as TPS) and the light stage approach proposed by Wilson et al. [Wilson et al. 2010] (referred to as WGP). During the experiments, eight input images are used for our approach and TPS. Six input images with spherical illumination are required for WGP. All input images are captured by two DSLR cameras with a resolution of $5496 \times 3670$ pixels. The second camera is only needed by our approach for estimating the global scalar to scale the initial reconstruction to the correct dimensions after normal integration (as described in Section 5).

\subsection{Synthetic Data}



Figure 3: A synthetic planar surface with six concave/convex grooves

Table 1: The RMSE (mm) of the estimated depth map to the ground truth depth map for each groove

\begin{tabular}{ccccc}
\hline Groove & Initial & Direct & First Iter. & Third Iter. \\
\hline 1 & 6.57 & 2.17 & 1.66 & 1.37 \\
2 & 6.53 & 3.92 & 2.16 & 1.86 \\
3 & 5.75 & 2.11 & 1.44 & 1.37 \\
4 & 4.19 & 3.5 & 3.45 & 3.48 \\
5 & 3.35 & 1.8 & 1.43 & 1.41 \\
6 & 3.07 & 2.27 & 1.9 & 1.78 \\
\hline
\end{tabular}

In this subsection, we present the results of the experiments with three synthetic objects: Grooves, Bunny and Face. All objects have a perfect diffuse material and the scenes are rendered using the Arnold ray tracing renderer in Autodesk 3ds Max, which physically emulates global illumination effects, such as indirect light from neighboring surfaces and self-shadowing.

For all three approaches the images are rendered with the following illumination patterns: eight perfect point lights for TPS; six spherical illuminations generated from 2202 point lights for WGP; and eight area lights for our approach.

As the ground truth normals and depth maps are known, we can compute the mean angular error (MAE) in degrees for the estimated normal maps and the root-mean-square error (RMSE) for the depth maps (which are obtained by integrating the normal maps).

We first evaluate the Grooves object by our proposed approach. As shown in Fig. 3, the Grooves object is a planar surface with a total of six concave and convex grooves. Groove 1 to groove 4 are concave and groove 5 and 6 are convex. We compute a normal map and a corresponding depth map by normal integration. The RMSE values are given in Table 1. The Initial values are the RMSE after the initial normal map estimation from Section 4. All grooves have a relatively large error for the initial reconstruction, especially groove 1, which is the deepest and has the largest cross-sectional area. Column Direct contains the RMSE values after considering only the first sum (direct light) of Eq. (10). Columns First Iter. and Third Iter. state the RMSE after the first and third iteration of the iterative optimization from Section 5 using the complete Eq. (10) (direct and indirect light). 


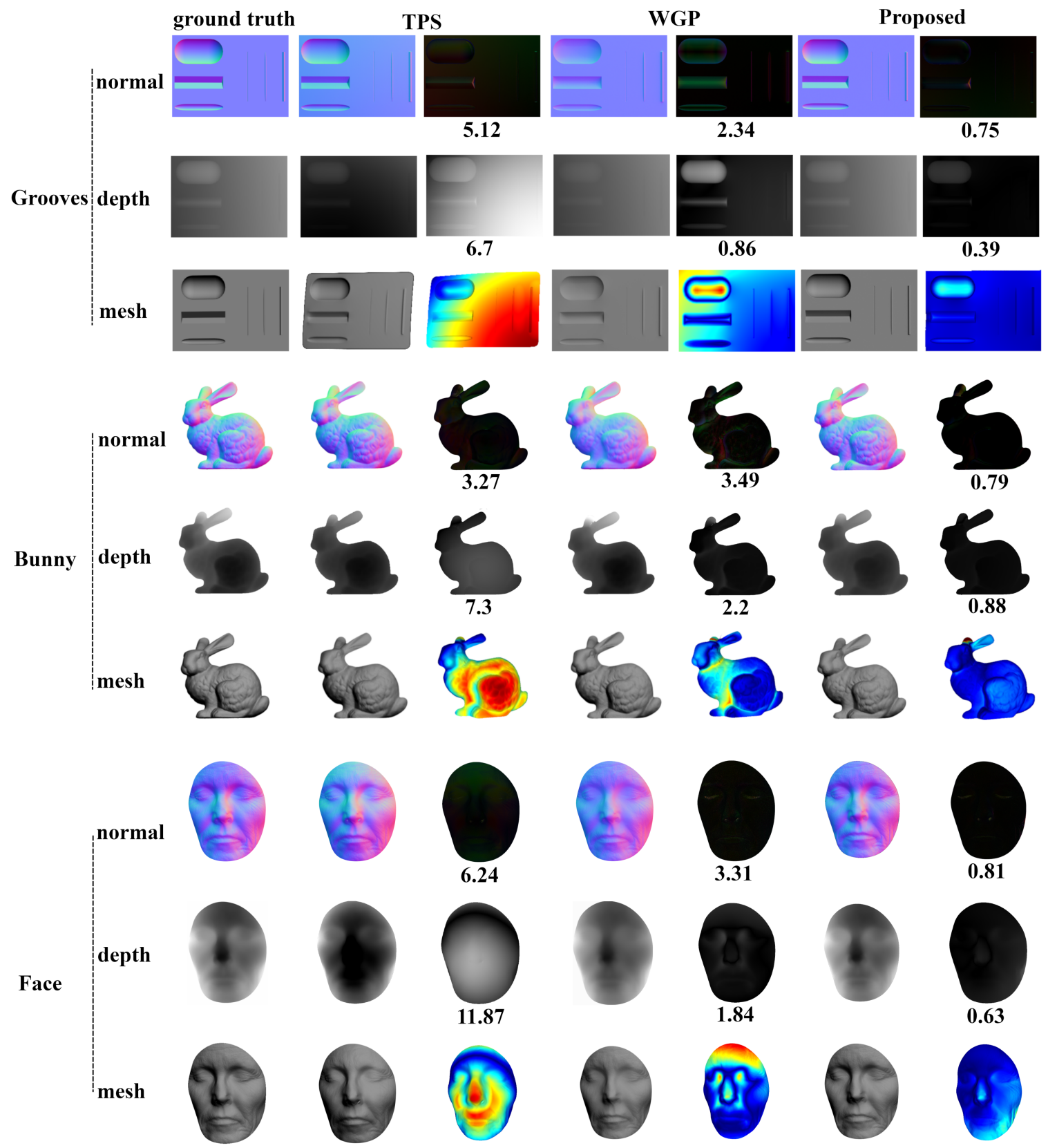

Figure 4: Estimated results and error comparison for the synthetic datasets using different approaches

Figure 4 shows the estimated results for the three synthetic objects by our approach and the other two approaches (TPS and WGP). The leftmost column contains the ground truth results. For each approach and each object, six images are shown: (first column) the normal map, the depth map, and the 3D reconstruction; (second column) the normal error map, the depth error map, and the reconstruction error map. The reconstruction error maps are colorized by pseudo-colors, where blue corresponds to a low error and red to a high error. The MAE in degrees and RMSE error values in millimeters are listed under the error maps. Overall, the results by TPS perform the worst. This can be explained because point lights are used for rendering instead of directional lights as assumed by Eq. (1). Consequently, the estimation of the normals is biased, the $3 \mathrm{D}$ reconstruction obtained from normal integration is skewed, and large errors can be observed. WGP produces much better results than TPS but still contains errors in the concave areas. These errors are produced from self-occlusion and interreflections that are not compensated. Our approach shows the best results for all synthetic 
experiments. However, it is important to note, that for TPS and WGP a similar compensation for nearby light sources, occlusion, and interreflection could be performed (but this is not part of the published algorithms).

\subsection{Real-world Data}

For the real-world experiments, we use a many-lights illumination hardware that was built from 2202 individual LEDs (shown in Fig. 5). Additionally, we placed eight area lights in the same setup.

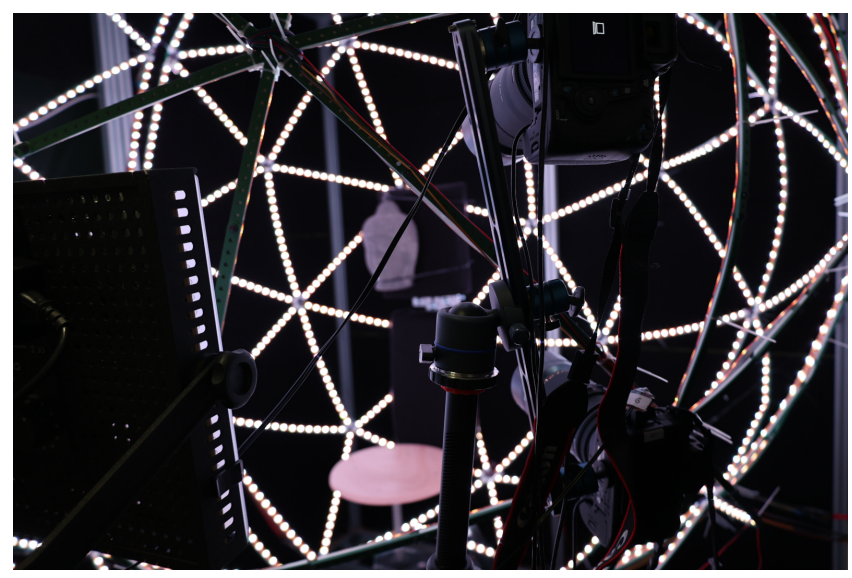

Figure 5: A many-lights illumination hardware and eight area lights are used subsequently for the same object

Afterwards, we captured the images for the six spherical gradients illuminations using the many-lights hardware as well as eight images for the different area light illuminations. The camera position and settings were not changed for the two capture sessions. Also, the captured object was not moved.

Figure 6 shows a comparison of the normal estimation with WGP and our approach. The normal maps are to some extend similar, but it is obvious that the normal maps from our approach contain less noise. This can be explained by the higher illuminance produces by the area lights compared to the many-lights illumination. Though the ISO settings on the cameras were not changed, higher luminance values were stored in the captured RAW images, which lead to less noise in the reconstructed normals. If the initial reconstruction and the final reconstruction by our approach is compared the surface features become more accentuated.

To evaluate further, we captured a reference object with two concave 45 degree grooves with a depth of 5.0 and 2.5 millimeters in the same setup. Fig. 7 shows the results for WGP and our approach. The reference object is much smaller than the complete capturing space and only a cropped area of the original image is shown in the figure. It can be clearly seen that WGP underestimates the depth of the grooves and the grooves do not have a sharp angle at their center. The mean angular error inside the groove is 25.44 degrees. In contrast, with our approach, the shape of the grooves is more similar to the reference object. The mean angular error is reduced to 13.1 degrees.

\section{CONCLUSION}

This paper shows that area lights can be used to generate normal maps using photometric stereo. Standard off-the-shelf area lights are easy to obtain, mount, and transport to different locations. Another advantage is their higher illuminance compared to point lights, which results in an improved signal-to-noise ratio and/or faster shutter speed.

A limitation of the current approach is that it is only evaluated for objects with continuous depth and diffuse reflectance in our experiments. Furthermore, it was assumed that the LED area lights can be modeled as perfect Lambertian emitters.

The major disadvantage of using area lights is that more complex light transport equations must be evaluated with ray tracing techniques. Our CUDA ray tracing implementation requires approximately 1 to 2 hours depending on the target area and the graphics card. However, it should be possible to reduce the computation time if we would use the ray tracing acceleration hardware that is available in recent GPUs (which we plan to evaluate in future work).

In contrast, using either spherical gradients illumination in a many-lights setup or traditional photometric stereo with point lights leads to simpler equations that are fast to evaluate. However, as shown in our experiments, these simple equations neglect nearby light sources, occlusion, and interreflections. Consequently, if the task is to compute the unbiased normals, similar solutions to our approach (that evaluate the true light transport with ray tracing) would be required for these methods as well.

In conclusion, if normal maps are required for movie production or special effects, fast and approximate results are currently best obtained with many-lights setups. But if a higher computational effort is acceptable, area lights are applicable for photometric stereo as well. They are easy to assemble and transport, and can produce a high illuminance which results in low image noise and fast shutter speeds.

\section{REFERENCES}

James J. Clark. 2006. Photometric Stereo with Nearby Planar Distributed Illuminants. In Third Canadian Conference on Computer and Robot Vision (CRV 2006), 7-9 Fune 2006, Quebec City, Canada. IEEE Computer Society, 16. https://doi.org/10.1109/ CRV.2006.55

James J. Clark. 2010. Photometric stereo using LCD displays. Image Vis. Comput. 28, 4 (2010), 704-714. https://doi.org/10.1016/j.imavis.2008.10.011

Paul Debevec. 2012. The Light Stages and Their Applications to Photoreal Digital Actors. In SIGGRAPH Asia Technical Briefs, November 26-27, 2012, Singapore.

Nathan Funk and Yee-Hong Yang. 2007. Using a Raster Display for Photometric Stereo. In Fourth Canadian Conference on Computer and Robot Vision (CRV 2007), 28-30 May 2007, Montreal, Quebec, Canada. IEEE Computer Society, 201-207. https: //doi.org/10.1109/CRV.2007.66

Martin P. Grochulla, Thorsten Thormählen, and Hans-Peter Seidel. 2011. Using Spatially Distributed Patterns for Multiple View Camera Calibration. In Computer Vision/Computer Graphics Collaboration Techniques - 5th International Conference, MIRAGE 2011, Rocquencourt, France, October 10-11, 2011. Proceedings (Lecture Notes in Computer Science, Vol. 6930), André Gagalowicz and Wilfried Philips (Eds.). Springer, 110-121. https://doi.org/10.1007/978-3-642-24136-9_10

Aaron Hertzmann and Steven M. Seitz. 2005. Example-Based Photometric Stereo: Shape Reconstruction with General, Varying BRDFs. IEEE Trans. Pattern Anal. Mach. Intell. 27, 8 (2005), 1254-1264. https://doi.org/10.1109/TPAMI.2005.158

Katsushi Ikeuchi. 1981. Determining Surface Orientations of Specular Surfaces by Using the Photometric Stereo Method. IEEE Trans. Pattern Anal. Mach. Intell. 3, 6 (1981), 661-669. https://doi.org/10.1109/TPAMI.1981.4767167

Miao Liao, Xinyu Huang, and Ruigang Yang. 2011. Interreflection removal for photometric stereo by using spectrum-dependent albedo. In The 24th IEEE Conference on Computer Vision and Pattern Recognition, CVPR 2011, Colorado Springs, CO, USA, 20-25 June 2011. IEEE Computer Society, 689-696. https://doi.org/10.1109/CVPR. 2011.5995343 

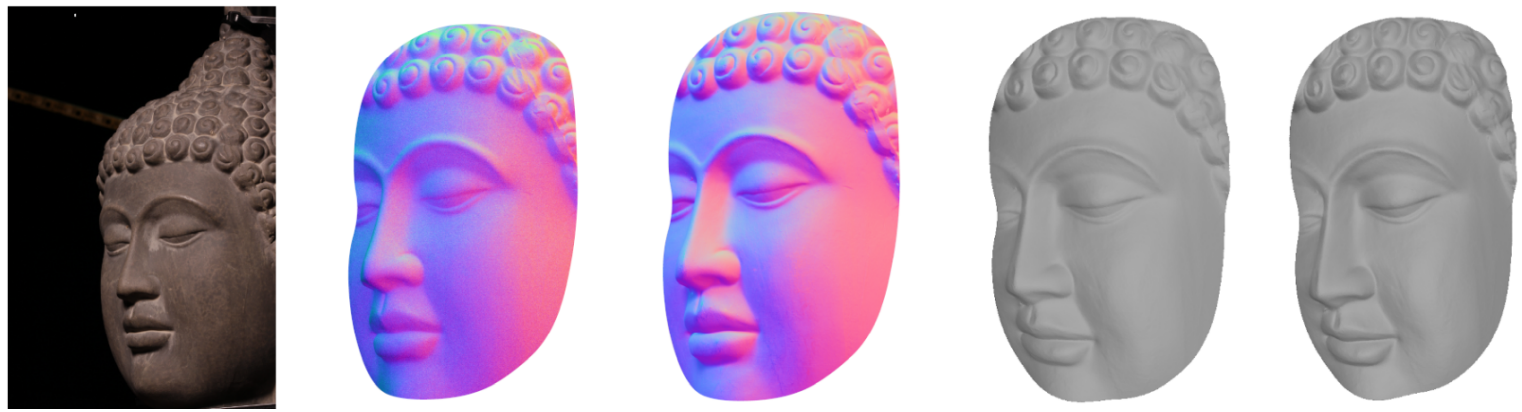

Figure 6: Comparison between WGP and our approach for a Buddha head. From left to right: input image, normal map for WGP, normal map for our approach, initial 3D reconstruction for our approach, final 3D reconstruction for our approach
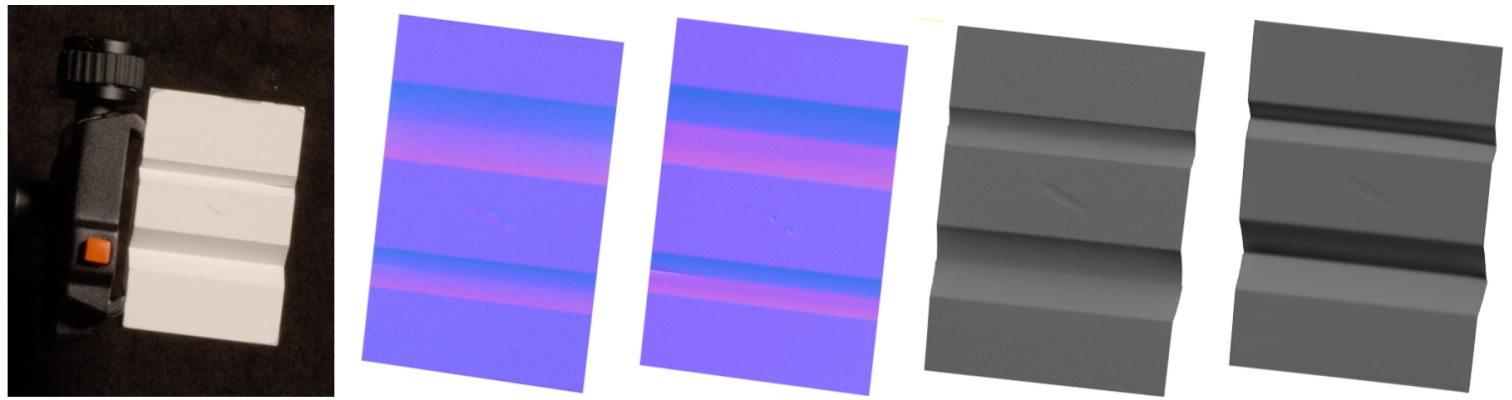

Figure 7: Comparison between WGP and our approach for a real-world reference object. From left to right: Cropped region from the input image, normal map for WGP, normal map for our approach, 3D reconstruction for WGP, 3D reconstruction for our approach

Chao Liu, Srinivasa G. Narasimhan, and Artur W. Dubrawski. 2018. Near-light photometric stereo using circularly placed point light sources. In 2018 IEEE International Conference on Computational Photography, ICCP 2018, Pittsburgh, PA, USA, May 4-6, 2018. IEEE Computer Society, 1-10. https://doi.org/10.1109/ICCPHOT.2018.8368465

Fotios Logothetis, Roberto Mecca, and Roberto Cipolla. 2017. Semi-Calibrated Near Field Photometric Stereo. In 2017 IEEE Conference on Computer Vision and Pattern Recognition, CVPR 2017, Honolulu, HI, USA, Fuly 21-26, 2017. IEEE Computer Society, 4521-4530. https://doi.org/10.1109/CVPR.2017.481

Wan-Chun Ma, Tim Hawkins, Pieter Peers, Charles-Félix Chabert, Malte Weiss, and Paul E. Debevec. 2007. Rapid Acquisition of Specular and Diffuse Normal Maps from Polarized Spherical Gradient Illumination. In Proceedings of the Eurographics Symposium on Rendering Techniques, Grenoble, France, 2007. 183-194. https://doi. org/10.2312/EGWR/EGSR07/183-194

Yvain Quéau, Bastien Durix, Tao Wu, Daniel Cremers, François Lauze, and Jean-Denis Durou. 2018. LED-Based Photometric Stereo: Modeling, Calibration and Numerical Solution. F. Math. Imaging Vis. 60, 3 (2018), 313-340. https://doi.org/10.1007/s10851017-0761-1

Yvain Quéau, Richard Modrzejewski, Pierre Gurdjos, and Jean-Denis Durou. 2016. A full photometric and geometric model for attached webcam $/$ matte screen devices. Signal Process. Image Commun. 40 (2016), 65-81. https://doi.org/10.1016/j.image. 2015.11.006
Hiroaki Santo, Michael Waechter, and Yasuyuki Matsushita. 2020. Deep Near-Light Photometric Stereo for Spatially Varying Reflectances. In Computer Vision - ECCV 2020 - 16th European Conference, Glasgow, UK, August 23-28, 2020, Proceedings, Part VIII (Lecture Notes in Computer Science, Vol. 12353), Andrea Vedaldi, Horst Bischof, Thomas Brox, and Jan-Michael Frahm (Eds.). Springer, 137-152. https: //doi.org/10.1007/978-3-030-58598-3 9

Yoav Y. Schechner, Shree K. Nayar, and Peter N. Belhumeur. 2003. A Theory of Multiplexed Illumination. In 9th IEEE International Conference on Computer Vision (ICCV 2003), 14-17 October 2003, Nice, France. IEEE Computer Society, 808-815. https://doi.org/10.1109/ICCV.2003.1238431

Zhao Song, Zhan Song, Juan Zhao, and Feifei Gu. 2020. Micrometer-level 3D measurement techniques in complex scenes based on stripe-structured light and photometric stereo. Optics Express 28(22) (2020), 32978-33001.

Cyrus A. Wilson, Abhijeet Ghosh, Pieter Peers, Jen-Yuan Chiang, Jay Busch, and Paul E. Debevec. 2010. Temporal upsampling of performance geometry using photometric alignment. ACM Trans. Graph. 29, 2 (2010), 17:1-17:11. https://doi.org/10.1145/ 1731047.1731055

Robert J. Woodham. 1980. Photometric method for determining surface orientation from multiple image. Optical Engineering 19, 1 (1980), 139-144.

Wuyuan Xie, Ying Nie, Zhan Song, and Charlie C. L. Wang. 2019. Mesh-Based Computation for Solving Photometric Stereo With Near Point Lighting. IEEE Computer Graphics and Applications 39, 3 (2019), 73-85. https://doi.org/10.1109/MCG.2019. 2909360 